\title{
Public Health Research Ethics and Clinical Research Ethics. How we differentiate?
}

\section{Zoheb Rafique}

Department of Community Medicine, Liaquat University of Medical \& Health Sciences (LUMHS), Jamshoro, Pakistan

Email: scorpionzoheb@hotmail.com

\begin{abstract}
This article talks about both clinical research ethics and public health research ethics. Clinical research ethics are defined as set of relevant ethics considered necessary for the conduct of clinical trials in field of the clinical research. While public health research ethics is mainly aimed at finding out what is best for the communities and the populations rather than the individuals. Research ethics is mainly focused on the protection of individual participants and some of the research norms and accepted principles may be challenging when applying in some of the research contexts.
\end{abstract}

Key words: Public Health; Research Ethics; Clinical Research Ethics

Introduction: The field of biomedical ethics arose in late 1960s in response to some emerging ethical dilemmas and issues of that era. This field for many years focused on dilemmas generated by the high technology medicine, rather than on the issues of the population health and the ethical problems of public health programs. The discipline Bioethics received the initial stimulus from abuses and issues of human subject's research, also the emergence of patient's rights movement, and the drama of high technology medicine. The Research involving human subjects has often been a central ethical issue and problem for the biomedicine for at least hundred (100) years now, and particularly since the World War 2. Just as the public health is broad in the scope, the range of the ethical issues in this field is uncommonly wide (1). In past twenty (20) years, the research base of the clinical ethics has gained tremendous strength appreciably. However the main research opportunities didn't come under broad heading of the clinical ethics, but instead through the specific programs such as human genome project and end of life movement (2). In this paper, I will discuss the differences between public health research ethics and clinical research ethics.

Discussion: The Public health research ethics include ethics regarding the community participation in the research, while the clinical research ethics include the ethics regarding the clinical patient research. This is major difference between these two scenarios, now I will discuss it in detail. Most of the research has focused on the clinical and the experimental medicine (efficacy, safety and the mechanism of action, and also regulatory issues regarding general neglect of the public health dimensions. The Public health ethics, which is defined as identification, analysis, and the resolution of the ethical problems occurring in the public health practice and also public health 
research. It has different domains than those of the medical ethics. The ethical concerns in the public health often relate to dual obligations of the public health professionals to apply and acquire the scientific knowledge that aimed at restoring and also protecting public health while respecting the individual autonomy. Ethics in the public health involves interplay between safeguarding welfare of individual, as in medicine, and also the public health goal of protecting public welfare. Some other ethical concerns in the public health relate to need to ensure just and fair distribution of the public health resources. The Public health ethics has broad scope that includes the ethical and the social issues arising in the health promotion and the disease prevention, the epidemiological research, and also public health practice. The main professional roles of the epidemiologists are design and the conduct of the scientific research and public health application of the scientific knowledge. This includes reporting the research results and also maintaining and promoting the health in communities. It also functions in carrying out the professional roles. The epidemiologists often encounter many ethical issues and some concerns that require the careful consideration. Many of those issues have been highlighted and addressed in literature on ethics in the epidemiology and public health including the ethics guidelines. The ethical and the professional norms in the epidemiology have also been clarified in the ethics guidelines for the epidemiologists and also the public health professionals. The Ethics guidelines such as those developed for Industrial Epidemiology Forum, International society for the Environmental
Epidemiology, and American college of the Epidemiology also provide useful accounts of the epidemiologists' obligations to the research participants, employs, society, and colleagues (3). The Epidemiological studies can provide the descriptive data that can lead scientists in the future to develop some interventions that can result in the reduction in morbidity and mortality; the health education program can be one of the multiple interventions that together reduce the risks and ill health. The argument here, however, is that the public health programs, studies, or interventions, must be designed with the awareness of relationship between that program and ultimate reduction in the morbidity and mortality. The Public health programs may result in high employment, as well as some less tangible benefits such as coalition building and strengthening of the communities. Today, the public health practitioners use some tools in addition to the epidemiology to register their work, still aiming primarily on community wide. It also typically uses prospective methods and approaches to improve health. In addition, the practitioners investigate the outbreaks, provide health education, conduct contact tracing, and some other preventive interventions, and also organize research related to the public health (4). The Public health agencies require the identifiable health information for conducting different public health activities. The increasing number of the functions, including the public health surveillance, and outbreak and incident investigations and program implementation, and some direct health services, such as the clinical public health activities and services and the research, maintenance, and the 
storage of the personal health information. The Successful execution of all these functions depends on the data quality and the accessibility. Heightened security is very necessary and paramount to maintain the public confidence; also good health care and it depends on the patients providing the accurate and sensitive information to their care providers in a very timely manner. Placing restrictions on the data acquisition, use, and the disclosure also poses some risks, particularly if those restrictions impede acquisition of the key surveillance data, which would otherwise be used to prevent the disease, investigate the causation, and enable the interventions to protect the exposed population. Additionally, electronic data could potentially permit real time public health surveillance and also can facilitate the faster emergency response (5). Advances in the science, technology and the biomedical research have pushed the boundaries of Belmont principles and stimulating the need for the communities to be involved in informed consent process. Changes in the Food and the Drug Administration regulations allow the waivers of the informed consent in life threatening emergencies. The rights of the unconscious participants are assumed to be accorded degree of the protection through mechanism of the "Community Consultation" which requires the prior consultation by the investigators and the institutional review boards with the community representatives and public disclosure to affected community both before and after that research (6). Now I will discuss the clinical research ethics, and we will see how it is different from public health research ethics. Taking into account the sound and the increasing emphasis of recent years that the experimentation in man must precede the general application of the new procedures in the therapy, and also there is reason to fear that these requirements and the resources might be greater than supply of the responsible investigators. Medical schools and the university hospitals are increasingly dominated by the investigators. Every young man knows that he will never be promoted to some tenure post, and to a professorship in a major medical school, unless he has proved himself as an investigator. If the ready availability of the money for conducting the research is added to this fact, one can see how great the pressure is on the ambitious young physicians (7). A taxonomy was developed for the clinical ethics research, based on the method rather than the clinical area. This divided research in different terms of whether it used theoretical or any empirical methods. First, we will see the theoretical methods of the clinical ethics research. Philosophy (e.g., How should the decisions on setting the priorities be made legitimate and also fair?). Law (e.g., what practices in setting the priorities in regional health authority might constitute discrimination?). Policy (e.g., what policy should the governments follow in funding the new technologies in medicine?). Now let's see the empirical methods of clinical ethics research. Social Sciences (e.g., how do the regional health authorities in the developing countries make the decisions on setting the priorities?). Decision analysis (e.g., How do you trade-off considerations of equity and efficiency in the decisions on setting priorities?). Clinical epidemiology (e.g., what are the criteria used to allocate the liver transplant?). Health services research 
(e.g., how does the delivery of the cardiac surgery vary by patient gender and ethnicity?). Within empirical research, there is some growing recognition that the quantitative methods alone are not adequate. Since many of the phenomena examined by the ethics researchers are deeply entwined into fabric of professions, organizations, and the human lives, qualitative methods have begun to play an important role. For example, one investigator performed the observational research on how physicians discuss do-notresuscitate orders and also advance care planning. The role of the qualitative methods is increasing to include not only the content analysis but also grounded theory, the ethnography, and the case study designs. When we review the field of the clinical ethics a decade from now, we hope that the focus will be shifted from the ethics courses, committees and the consultants to an understanding on the part of most physicians and medical students that ethics is an inherent and inseparable part of the good clinical medicine. We hope that clinical ethics will achieve its rightful place at the interstices of relations between the patients who are sick and physicians who profess to be able to heal and comfort them. Clinical ethics has made progress towards this vision in the past some years. The challenge remains for the research to become a mainstream concern for the funding agencies around the World.

Conclusion: In conclusion, it is stated that although public health research and clinical research are different from each other, but ethical dilemmas are faced by both and also they are same in many situations. While considering the public health research ethics, the researcher must show respect for culture of the community, also take community input on the protocol development, and ensure that research is useful to community, and should respect the knowledge and experience of the community, and ensure that the informed consent is correctly taken before starting any of the research (8). While considering the clinical research ethics, two components are most important. The first being the informed consent, and the statement that informed consent has been obtained has very little meaning unless the participant or his/her guardian is capable of understanding what is to be undertaken and all of risks/benefits are explained clearly. Secondly, there is more reliability provided by the presence of intelligent, informed, compassionate, conscientious, and responsible investigator.

\section{References:}

1. Daniel Callahan and Bruce Jennings. Ethics and Public Health: Forging a Strong Relationship. American Journal of Public Health. 2002; 92(2): 169176.

2. Peter A Singer Et Al. Clinical Ethics Revisited. BMC Medical Ethics. 2001; 2:1.

3. Steven S Coughlin. Ethical issues in epidemiologic research and public health practice. Emerging Themes in Epidemiology. BioMed central. 2006; 3:16.

4. Nancy E. Kass. An Ethical Framework for Public Health. Public Health Matters.

5. Julie Myers Et Al. Privacy and Public Health at Risk: Public Health Confidentiality in the Digital Age. American Journal of Public Health. 2008; 98(5): 793801.

6. Sandra Crouse Quinn. Protecting Human Subjects: The Role of Community Advisory Boards. American Journal of Public Health. 2004; 94(6): 918-922.

7. Henry K. Beecher. Ethics and Clinical Research. The New England Journal of Medicine. 1966; 274(24): 1354-1360.

8. C. Weijer and E.J. Emanuel. Protecting Communities in Biomedical Research. Science. Policy Forum: Ethics. 2000; 289: 1142-1144 